
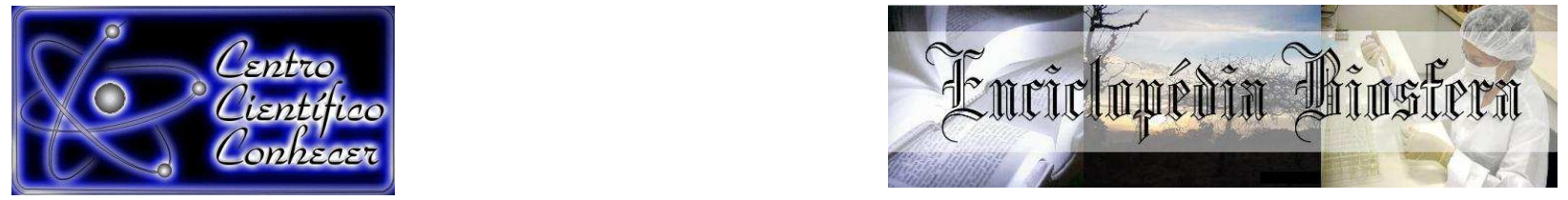

\title{
ECTOPARASITOS EM CÃES DE ÁREAS PERI-RURAIS DO MUNICÍPIO DE RIO BRANCO, ACRE, AMAZÔNIA OCIDENTAL
}

Juliana Milan de Aquino Silva ${ }^{1}$, Joelma de Faria Santos ${ }^{2}$, Marcia Sangaletti Lavina ${ }^{3}$, Antonio Pereira de Souza ${ }^{4}$, Soraia Figueiredo de Souza ${ }^{5}$

1 - Doutoranda do Programa de Pós Graduação em Sanidade e Produção Animal Sustentável na Amazônia Ocidental, Universidade Federal do Acre, Rio Branco, AC, Brasil. Email: juliana_milan@yahoo.com.br

2 - Discente de Graduação em Medicina Veterinária, Universidade Federal do Acre, Rio Branco, AC, Brasil.

3 - Docente do Curso de Medicina Veterinária, Universidade do Estado de Santa Catarina, Lages, SC, Brasil.

4 - Docente do Mestrado Profissional em Produção e Sanidade Animal, Instituto Federal Catarinense, Araquari, SC, Brasil.

5 - Docente do Curso de Medicina Veterinária, Universidade do Federal do Acre, Rio Branco, AC, Brasil.

Recebido em: 02/10/2017 - Aprovado em: 21/11/2017 - Publicado em: 05/12/2017 DOI: 10.18677/EnciBio_2017B26

\begin{abstract}
RESUMO
O presente trabalho estudou a caracterização de carrapatos, pulgas, piolhos e miíases em cães de áreas peri-rurais do município de Rio Branco, Acre. Os espécimes foram coletados por meio de inspeção visual e tátil do corpo dos animais, e posteriormente, colocados em frascos contendo etanol $96^{\circ} \mathrm{GL}$ e identificados sob microscópio e/ou estereomicroscópio. Foram examinados 100 cães com a presença de ectoparasitas, que na sua maioria eram sem raça definida (SRD). No que se refere ao gênero, 60 eram machos e 40 eram fêmeas. Destes, 14 eram jovens, 64 eram adultos e 22 eram idosos. Dos ectoparasitas identificados, 50 cães possuíam carrapatos (Rhipicephalus sanguineus e Amblyomma ovale); 71 animais estavam com pulgas (Ctenocephalides felis e Ctenocephalides canis); 8 cães apresentaram piolhos (Trichodectes canis e Heterodoxus spiniger) e dois miíase (Dermatobia hominis), sendo que $20 \%$ dos cães apresentaram parasitismo misto. Quanto a realização de tratamentos para ectoparasitas apenas $8 \%$ animais receberam alguma medida preventiva e/ou de combate a infestação. Com base nos resultados constatados, conclui-se que a pulga $C$. felis e o carrapato $R$. sanguineus constituíram as espécies de ectoparasitas predominantes em cães de áreas perirurais do município de Rio Branco, Acre.
\end{abstract}

PALAVRAS-CHAVE: Ctenocephalides felis; ectoparasitismo; Rhipicephalus sanguineus

\section{ECTOPARASITES IN DOGS OF PERI-RURAL AREAS OF RIO BRANCO MUNICIPALITY, ACRE, WESTERN AMAZON}


The present work investigated the characterization of ticks, fleas, lice and myiasis in dogs from peri-rural areas of the city of Rio Branco, Acre. The specimens were collected by means of visual and tactile inspection of the animals' body and then placed in flasks containing $96^{\circ} \mathrm{GL}$ ethanol and identified under a microscope and / or stereomicroscope. One hundred dogs were examined with the presence of ectoparasites, which were mostly were of a non-defined breed. Regarding the gender, 60 were males and 40 were females. Of these, 14 were young, 64 were adults and 22 were elderly. Of the ectoparasites identified 50 dogs had ticks (Rhipicephalus sanguineus and Amblyomma ovale); 71 animals had fleas (Ctenocephalides felis and Ctenocephalides canis); 8 dogs were infested with lice (Trichodectes canis and Heterodoxusspiniger) and two myiasis (Dermatobiahominis), and $20 \%$ of the dogs showed mixed parasitism signs. Regarding the performance of the treatments for ectoparasites only $8 \%$ of the animals received some preventive measure and / or to combat infestation. Based on the results, it was concluded that the $C$. felis flea and the $R$. sanguineus tick were the predominant species of ectoparasites in dogs from the peri-rural areas of the city of Rio Branco, Acre.

KEYWORDS: Ctenocephalides felis; ectoparasitism; Rhipicephalus sanguineus

\section{INTRODUÇÃO}

Ectoparasitas são artrópodes encontrados na pele ou nas camadas superficiais da mesma de diferentes espécies de animais (LEVINSON, 2014). Os cães podem ser parasitados por piolhos, ácaros produtores de sarna, pulgas, carrapatos, piolhos e larvas de moscas. O grau de severidade à saúde do hospedeiro é influenciado pela imunidade do mesmo e o tamanho da carga parasitária (DANTAS-TORRES; OTRANTO, 2014).

A presença de cães nos lares brasileiros está cada vez mais frequente. Domingues (2013) estimou esse crescimento em torno de 1,4 milhões de cães entre 2011 e meados de 2013, e segundo a Associação Brasileira da Indústria de Produtos para Animais de Estimação - ABINPET (2014), 91\% das rações para animais produzidas são específicas para cães. O aumento populacional de cães deve ser considerado dentro de programas de saúde pública, pois os ectoparasitas possuem ação espoliadora e são vetores de agentes de patogênicos para os cães e humanos (CASTRO; RAFAEL, 2006). Melo et al. (2016) verificaram que $15,40 \%$ dos Amblyomma ovale amostrados estavam infectados por Rickettsia sp. e alertaram sobre como os seres humanos estão potencialmente expostos à infecção por agentes bacterianos e protozoários transmitidos por carrapatos. Além disso, Babesia, Ehrlichia e Anaplasma são importantes e prevalentes hemoparasitoses em na clínica médica de pequenos animais (RIBEIRO et al., 2017).

Segundo Galvéz et al. (2017), cães de fazendas têm maior risco de infestação por pulgas do que cães de abrigos. Por isso, o conhecimento da epidemiologia das ectoparasitoses em cães de área rural é uma ferramenta importante para estratégias preventivas e de controle dos mesmos, visando a interrupção de ciclos de diferentes enfermidades.

O clima do município de Rio Branco, Acre, é equatorial, quente e úmido, alternado por duas épocas distintas, sendo chuvosa de novembro a abril e seca de maio a outubro (GOVERNO DO ACRE, 2015), o que o torna propício para algumas espécies de ectoparasitas (TAYLOR, 2010). Além disso, não é incomum o contato dos cães de áreas rurais de Rio Branco com animais silvestres, outra população de 
hospedeiros. Ainda é desconhecida a ocorrência de ectoparasitas permutados entre animais silvestres e cães de caça (DANTAS-TORRES; OTRANTO, 2014). Não foram encontrados estudos sobre a ectofauna parasitária em caninos domésticos no município de Rio Branco, Acre, Amazônia Ocidental, desta forma, o objetivo deste trabalho foi avaliar a epidemiologia de carrapatos, pulgas, piolhos e larvas de miíases em cães oriundos na área peri-rural.

\section{MATERIAL E MÉTODOS}

As coletas foram realizadas em propriedades peri-rurais do município de Rio Branco, AC, localizadas ao longo da Rodovia Transacreana (AC-90) latitude 1000'40.8'S e longitude 67ํ51'54.8' W, na Vila Custódio Freire (BR-364)

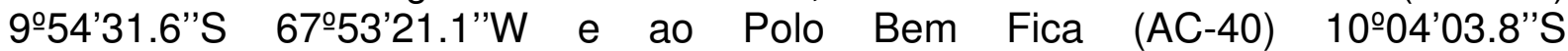
67ำ4'56.8' W, ou próximas a estrada para Porto Acre (AC-10) 955'07.8'S 67ํํ'23.6' $\mathrm{W}$, principalmente durante o período de estiagem. Os cães foram examinados na presença de seus respectivos tutores, sob adequada contenção física. Dos 100 cães positivos para ectoparasitas foram coletadas espécimes de carrapatos, pulgas, piolhos ou miíases.

Cada animal foi submetido a inspeção direta, afastando-se os pelos, inspecionando orelhas, entre o dígitos e lugares quentes do corpo como região das axilas e abdômen. Ao detectar o ectoparasita, este foi coletado e acondicionado em frasco de vidro hermeticamente fechado, contendo álcool $96^{\circ}$ devidamente identificado. Os dados sobre sexo, idade e realização ou não de tratamento para controle de ectoparasitas foram anotados em fichas individuais.

Todas a amostras coletadas foram encaminhadas para Laboratório de Análises Clínicas e Parasitologia da Unidade de Ensino e Pesquisa em Medicina Veterinária Mário Alves Ribeiro da Universidade Federal do Acre da UFAC, onde foram identificados os gêneros e espécies dos ectoparasitas coletados. Para identificação utilizou-se microscópio óptico ou estereomicroscópio, e tomou-se referência pela chave taxonômica elaborada de acordo com a morfologia descrita por Foreyt (2005) e Taylor et al. (2010).

Realizou-se um estudo prospectivo descritivo, onde os dados gerados foram tabulados em planilha Excel e analisados observando-se as frequências: a) das espécies ou gêneros dos ectoparasitas na população total amostrada; b) de acordo com as seguintes características dos caninos examinados quanto: ao sexo (macho ou fêmea); a idade (jovem abaixo de 2 anos, adulto de 2 anos a 7 anos e 11 meses, idoso acima de 8 anos) e se recebeu algum tipo de tratamento ou não para ectoparasitas; c) tipo de infestação podendo ser unitária (uma espécie ou gênero) ou mista (duas ou mais espécies e/ou gênero). Este estudo foi conduzido sob aprovação do Comitê de Ética no Uso de Animais - CEUA/UFAC (protocolo no 25/2014).

\section{RESULTADOS E DISCUSSÃO}

Espécimes de ectoparasitas foram coletados em 100 cães positivos para ectoparasitos, sendo eles $98 \%$ mestiços e $2 \%$ de raças definidas, representados por 60 machos e 40 fêmeas. As idades variaram entre dois meses e dez anos de vida, sendo $14 \%$ de jovens ( $<1$ ano de idade), $64 \%$ de adultos ( 1 a 7 anos de idade) e $22 \%$ de idosos ( $\geq 8$ anos de idade). 
Dos ectoparasitas identificados, $50 \%$ dos cães possuíam carrapatos (Rhipicephalus sanguineus e/ou Amblyomma ovale); $71 \%$ estavam com pulgas (Ctenocephalides felis e/ou Ctenocephalides canis); $8 \%$ apresentaram piolhos (Trichodectes canis e/ou Heterodoxus spiniger) e $2 \%$ tinham larva de Dermatobia hominis, sendo que alguns cães apresentaram parasitismo misto (20\%).

Dentro da população total amostrada de 100 cães a frequência dos espécimes de ectoparasitas de acordo com o acometimento por gênero estão representadas na Figura 1.

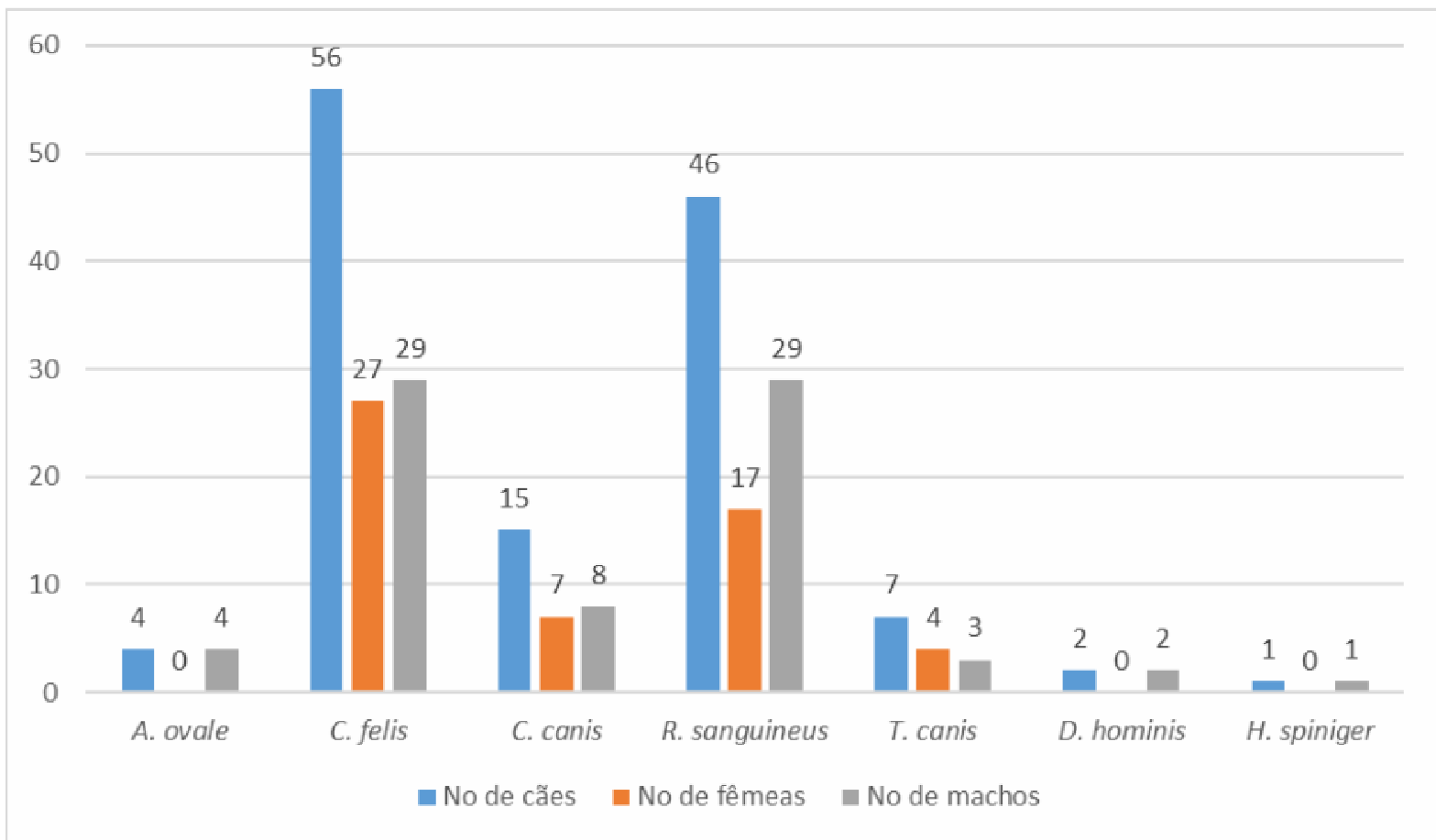

FIGURA 1. Frequências de diferentes espécies de ectoparasitas que acometiam cães em áreas na zona peri-rural de Rio Branco, AC, de acordo com o gênero do hospedeiro.

Os resultados obtidos neste estudo mostraram que cães machos abrigaram mais ectoparasitas que as fêmeas. Isto pode ser explicado pela proximidade entre algumas propriedades rurais, que pode facilitar o agrupamento de cães machos durante o período de estro em uma fêmea. Segundo Hoogland (1998) a cópula é um fator que aumenta a exposição deste gênero a doenças e parasitas. Esse resultado corrobora com Cardoso et al. (2015) que verificaram, num intervalo de 10 anos, maior número de machos com infestação ectoparasitária que cadelas.

No que concerne à idade, a prevalência de infestação por ectoparasitas foi maior entre cães de dois a sete anos de idade $(64 ; 64 \%)$ quando comparados aos cães com menos de dois anos (14; $14 \%$ ) e mais de oito anos de idade $(22 ; 22 \%)$. O que difere dos resultados observados por Chee et al. (2008), os quais relataram maior incidência de ectoparasitas em cães com menos de 1 ano $(66,7 \%)$ e de Bessas et al. (2016) que dentre os 11 cães com ectoparasitas e positivos para infecções bacterianas na Argélia, aproximadamente $82 \%$ eram de idade inferior a dois anos.

Quanto a realização de tratamentos para ectoparasitas apenas $8 \%$ dos animais receberam alguma medida preventiva e/ou de combate a infestação, sendo 
que a maioria dos tutores (92\%) não faziam o controle de ectoparasitas em seus cães. Este resultado é condizente com afirmação de Dantas-Torres e Otranto (2014), sobre o baixo nível de consciência e de compliaence por parte dos proprietários de animais de áreas rurais, no que diz respeito ao controle de ectoparasitas. A decisão do uso ou não de antiparasitários pode diferir entre regiões do país, segundo o estudo de Figueredo et al. (2017), houve grande variação no número de proprietários que relataram fazer uso desses medicamentos de acordo com o local onde residiam, sendo que 6\% em Pernambuco e 60\% no Distrito Federal administravam antiparasitários em seus cães.

A partir dos dados coletados, pode-se inferir que piolhos ( $T$. canis e $H$. spiniger) e miíases ( $D$. hominis) acometeram os cães em menor frequência que pulgas e carrapatos. A ocorrência de pulgas ( $C$. felis e $C$. canis) e carrapatos $(R$. sanguineus e $A$. ovale) em animais que receberam algum tipo de tratamento, pode ser por tratamento incorreto quanto a forma e/ou tempo de aplicação; quanto ao tipo de medicamento (eficiência ou ação), e pelo fato do tratamento ser realizado apenas no animal, não havendo preocupação com o controle dos ectoparasitas no ambiente. Matos et al. (2015) reportaram que apesar da maioria dos proprietários, usarem medicamentos antiparasitários em seus cães e gatos, a aplicação é realizada em intervalos irregulares, resultando em tratamento ineficaz.

Dentre os animais que apresentaram monoparasitismo (80\% do total), observou-se que $57,5 \%$ destes animais estavam parasitados por pulgas, $41,25 \%$ por carrapatos e $1,25 \%$ por piolhos. O predomínio de pulgas $(73,3 \%)$ em relação aos carrapatos $(63,3 \%)$ também foi observado em 104 cães examinados em Juiz de Fora, Minas Gerais (RODRIGUES et al., 2001). Baixos níveis de infestações por piolhos também foram relatados anteriormente por Chee et al. (2008) na República da Coreia e Costa et al., (2013) no Estado do Maranhão.

Apesar de não ter sido um parâmetro avaliado no presente estudo, a época do ano pode ter influenciado na ocorrência de infestação por ectoparasitas, como observado por Paz et al. (2015), que verificaram maior prevalência nas infestações por de $C$. felis felis em meses de menor precipitação. A baixa incidência de cães parasitados por $D$. hominis pode ser devido ao fato das coletas terem sido realizada, em sua maioria, na época seca, o que desfavorece o ciclo biológico deste parasita, como foi observado por Cramer-Ribeiro et al. (2001), que relataram maior ocorrência de miíases em cães no período chuvoso. Ou por se tratarem de animais não negligenciados. Barbosa e Vasconscelos (2015) associam as miíases de cães e gatos com o abuso e negligência de seus proprietários, uma vez que as espécies de moscas tendem a se beneficiar de indivíduos subnutridos, com hematomas e em condições precárias de higiene.

Em $20 \%$ dos cães, foram identificados casos de poliparasitismo. Torres et al. (2004), Chee et al. (2008) e Paz et al. (2017) também encontraram maior frequência de animais apresentando apenas uma espécie de ectoparasita, sendo $95,4 \%, 83 \%$ e $80 \%$, respectivamente. Os cães avaliados em áreas peri-rurais do município de Rio Branco, apresentaram maior associação de pulgas e carrapatos (Figura 2). 


\section{\% CÃES PARASTADOS}

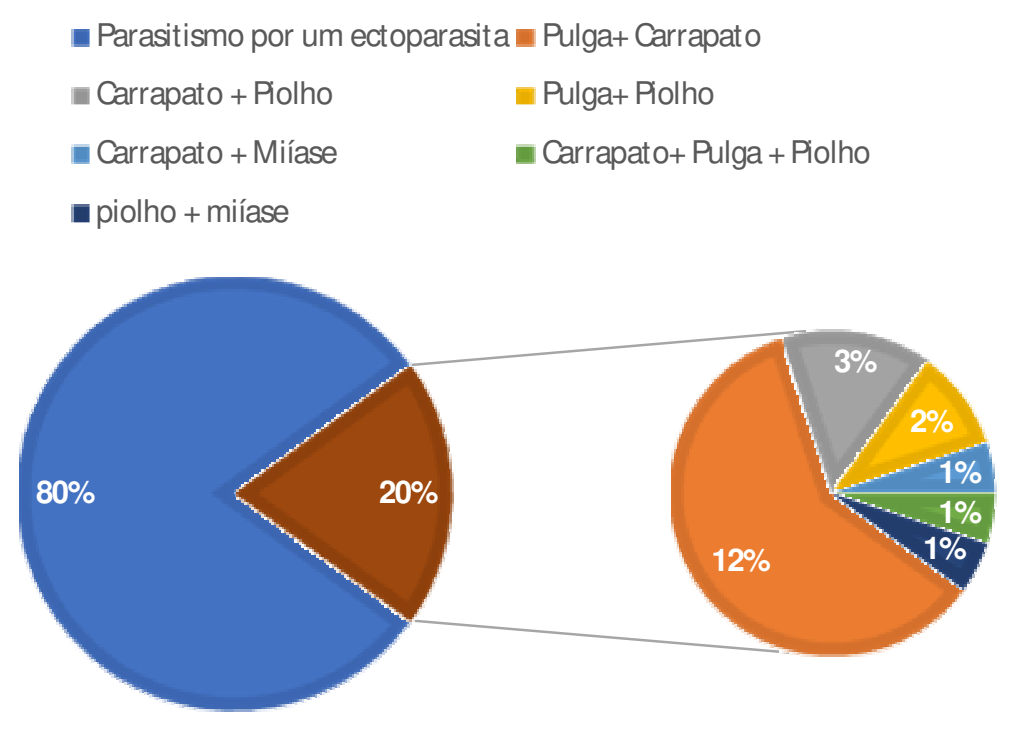

FIGURA 2. Percentual de ocorrência isolada ou simultânea de infestação por carrapatos, pulgas e piolhos em cães de área peri-rural do município de Rio Branco, Acre.

Torres et al. (2004) também relataram em seus estudos as associações de pulgas e carrapatos como de maior ocorrência, dentro do poliparasitismo, seguida pelas de carrapatos com piolhos e de pulgas com piolhos, outras associações não foram relatadas por esses autores. Foram encontradas as seguintes associações de ectoparasitas (número de observações entre parênteses): C. felis + C. canis (8); R. sanguineus + C. felis (7); R. sanguineus + C. felis + C. canis (2); $R$. sanguineus $+C$. canis (2); $R$. sanguineus $+T$. canis (2); Amblyomma ovale + C. felis (1); C. felis + T. canis (2); R. sanguineus + C. felis + T. canis (1); $R$. sanguineus $+H$. spiniger (1); $R$. sanguineus $+D$. hominis (1) e $T$. canis e $D$. hominis (1).

As espécies encontradas e suas respectivas prevalências, estão representadas na Tabela 1.

TABELA 1. Ectoparasitas que infestaram cães de área peri-rural do município de Rio Branco, Acre.

Ectoparasita

\begin{tabular}{ccc}
\hline \multirow{2}{*}{ Carrapatos } & Rhipicephalus sanguineus & $47 \%$ \\
\cline { 2 - 3 } & Amblyomma ovale & $4 \%$ \\
\hline \multirow{2}{*}{ Pulgas } & Ctenocephalides felis & $56 \%$ \\
\cline { 2 - 3 } & Ctenocephalides canis & $15 \%$ \\
\hline \multirow{2}{*}{ Piolhos } & Trichodectes canis & $7 \%$ \\
\cline { 2 - 3 } Miíases & Heterodoxus spiniger & $1 \%$ \\
\hline
\end{tabular}

Neste estudo foram encontradas duas espécies de carrapato sendo a de maior prevalência a Rhipicephalus sanguineus sobre a de Amblyomma ovale, esse 
achado pode ser explicado em função de se tratar de regiões rurais próximas a área urbana; ou ser um indício que $R$. sanguineus esteja disseminado por todo 0 município de Rio Branco. Costa et al. (2017), relataram suspeita de disseminação de $R$. sanguineus no estado do Maranhão após os resultados de seus estudos revelarem o predomínio desse ectoparasita em cães rurais e urbanos de diferentes regiões do estado, contribuindo com Dantas-Torres (2010), que descreveu esse carrapato como o mais difundido no mundo, presente em áreas urbanas e rurais e ativos 0 ano todo em regiões tropicais, subtropicais e em algumas zonas temperadas.

Não foram encontrados estudos registrando a presença do Amblyomma ovale parasitando cães no Estado do Acre, podendo este ser o primeiro registro do mesmo. O parasitismo de Amblyomma ovale nos cães de áreas peri-rurais do munícipio de Rio Branco, AC, os predispõem a serem acometidos por hepatozoonose canina uma vez que Rubini et al. (2009) afirmam que $A$. ovale é o vetor potencial de hepazoonose em cães rurais. Além do mais, Sousa et al. (2017) verificaram a alta prevalência de Hepatozoon entre carnívoros amostrados no Pantanal e a ocorrência de infestação de $A$. ovali em animais silvestres.

A espécie predominante de pulgas em todas as regiões foi Ctenocephalides felis, à medida que esporadicamente foi verificada Ctenocephalides canis. Neste estudo, quando comparado a ocorrência de cada espécie encontrada, esperava-se que cães rurais apresentassem parasitismo predominante por carrapatos, porém notou-se maior número de cães parasitados por $C$. felis, semelhantemente a Gracia et al. (2008), que relataram maior incidência de pulgas em cães de áreas rurais do que os de áreas urbanas. Como já citado anteriormente, este fato pode estar relacionado ao período de estiagem.

Dentre os piolhos, Trichodectes canis foi o mais encontrado. Este ectoparasita também tem sido encontrado em dessemelhantes regiões do Brasil parasitando cães, independente da área onde ele se encontra (RODRIGUES et al., 2001; COSTA et al., 2013), sugerindo que o tipo de ambiente onde os cães se encontram não tem interferência sobre a presença deste ectoparasita. Quanto à Heterodoxus spiniger, apesar da baixa porcentagem (1\%) de ocorrência, Bowman (2010) afirma que refere-se a um parasita com ampla difusão geográfica. TorresChable et al. (2017) relataram a infestação desse ectoparasita em 100\% dos cães domésticos amostrados, estes eram provenientes de famílias de vulnerabilidade social.

\section{CONCLUSÃO}

A pulga Ctenocephalides felis e o carrapato Rhipicephalus sanguineus constituíram as espécies de ectoparasitas predominantes em cães com infestação por carrapatos, pulgas, piolhos ou miíases de áreas peri-rurais do município de Rio Branco, Acre.

\section{REFERÊNCIAS}

ABINPET (Associação Brasileira da Indústria de Produtos para Animais de Estimação). ABINPET anuncia dados do mercado de pet food para 2014. Disponível em: <http://abinpet.org.br/imprensa/noticias/abinpet-anuncia-dados-domercado-de-pet-food-para-2014/>. Acesso em: 23 mai. 2015. 
BARBOSA, T. M.; VASCONCELOS, S.D. An updated checklist of myiasis-inducing Diptera species in livestock in Northeastern Brazil. Archivos de zootecnia, v. 64, n. 246, p. 187-190, 2015. Disponível em: <http://dx.doi.org/10.21071/az.v64i246.396>. doi: 10.21071/az.v64i246.396

BESSAS, A.; LEULMI, H.; BITAM, I.; ZAIDI, S.; AIT-OUDHIA, K.; et al Molecular evidence of vector-borne pathogens in dogs and cats and their ectoparasites in Algiers, Algeria. Comparative Immunology, Microbiology and Infectious Diseases, $\quad$ v.45, p.23-28, 2016. Disponível em: <https://doi.org/10.1016/j.cimid.2016.01.002>. doi: 10.1016/j.cimid.2016.01.002.

BOWMAN, D. D. Parasitologia Veterinária de Georgis. $8^{\underline{a}}$ ed. Barueri, SP: Manole, 2010. 432 p.

CARDOSO, I. R. S.; FILGUEIRA, K. D.; NASCIMENTO, J. O.; PEREIRA, J. S.; REIS-LIMA, R. K.; et al. Ectoparasitas na espécie canina: análise de dez anos. Revista de Educação Continuada em Medicina Veterinária e zootecnia do CRMV-SP, v. 13, n. 3, p.49-49, 2015. Disponível em:<http://revistas.bvsvet.org.br/recmvz/article/view/28262>. ISSN 2179-6645.

CASTRO, M. C. M.; RAFAEL J. A. Ectoparasitos de cães e gatos da cidade de Manaus, Amazonas, Brasil. Acta Amazonica, v.36, n.4, p.535-538, 2006. Dinponível em:<http://dx.doi.org/10.1590/S0044-59672006000400015>. ISSN:0044-5967.

CHEE, J. H.; KWON, J. K.; CHO, H. S.; CHO, K. O.; LEE, Y. J.;et al. A survey of ectoparasite infestations in stray dogs of Gwang-ju City, Republic of Korea. Korean Journal of Parasitology, v.46, n.1, p.23-27, 2008. Disponível em:<https://www.ncbi.nlm.nih.gov/pmc/articles/PMC2526298/>. doi: 10.3347/kjp.2008.46.1.23

COSTA, A. P.; SILVA, A. B.; COSTA, F. B.; XAVIER, G. S.; MARTINS, T. F.; et al. A survey of ectoparasites infesting urban and rural dogs of Maranhão state, Brazil. Jornal of Medical Entomology, v. 50, n. 3, p. 674-678, 2013. Disponível em:<https://doi.org/10.1603/ME12243>. doi:10.1603/ME12243.

COSTA, F. B.; COSTA, A. P. da; MORAES-FILHO, J.; MARTINS, T. F.; SOARES, H. S.; et al. Rickettsia amblyommatis infecting ticks and exposure of domestic dogs to Rickettsia spp. in an Amazon-Cerrado transition region of northeastern Brazil. PLOS ONE, $\quad$ v.12, $\quad$ n. $6, \quad 2017 . \quad$ Disponível em: <https://doi.org/10.1371/journal.pone.0179163>. doi: 10.1371/journal.pone.0179163

CRAMER-RIBEIRO, B. C.; SANAVRIA, A.; OLIVEIRA, M. Q.; SOUZA F. S.; ROCCO, F. S; et al. Inquérito sobre os casos de miíase por Cochliomyia hominivorax em cães da zona sul do município do Rio de Janeiro no ano 2000. Brazilian Journal of Veterinary Research and Animal Science, v.39, n.4, p.171-175, 2002. Disponível em:<http://dx.doi.org/10.1590/S1413-95962002000400003>. doi: 10.1590/S141395962002000400003. 
DANTAS-TORRES, F. Biology and ecology of the brown dog tick, Rhipicephalus sanguineus. Parasites \& Vectors, v. 3, n. 26, 2010. Disponível em:< http://doi.org/10.1186/1756-3305-3-26>. doi: 10.1186/1756-3305-3-26.

DANTAS-TORRES, F; OTRANTO, D. Dogs, cats, and humans in Brazil: opening the black box. Parasites \& Vectors, v.7, n. 22, 2014. Disponível em:<http://www.parasitesandvectors.com/content/7/1/22 >. doi: 10.1186/1756-3305$7-22$.

DOMINGUES, A. Brasil tem a segunda maior população de cães e gatos do mundo. Disponível em: <http://www.petrede.com.br/2013/animais/brasil-tem-asegunda-maior-populacao-de-caes-e-gatos-do-mundo/ > . Acesso em: 23 de maio de 2015.

FIGUEREDO, L. A.; SALES, K. G. da S.; DEUSTER, K.; POLLMEIER, M.; OTRANTO, D.; et al. Exposure to vector'borne pathogens in privately owned dogs living in diferente socioeconomic settings in Brazil. Veterinary Parasitology, v. 243. N. 30, p. 18-23, 2017. Disponível em:<https://doi.org/10.1016/j.vetpar.2017.05.020>. doi: 10.1016/j.vetpar.2017.05.020.

FOREYT, W. J. Parasitologia Veterinária: manual de referência. 5ed. Roca, 2005. 240p.

GÁLVEZ, R.; MONTOYA, A.; CHECA, R.; MARTíN, O.; MARINO, V. et al. Flea species infesting dogs in Spain: updated spatial and seasonal distribution patterns. Medical and Veterinary Entomology, v. 31, n.1, p 107-113, 2017. Disponível em: <http://onlinelibrary.wiley.com/enhanced/exportCitation/doi/10.1111/mve.12204>. doi: $10.1111 /$ mve.12204.

GOVERNO DO ACRE. 0 Estado. Disponível em: <http://www.ac.gov.br/wps/portal/acre/Acre/estado-acre >. Acesso em: 22 de maio de 2015.

GRACIA, M. J.; CALVETE, C.; ESTRADA, R.; CASTILLO, J. A.; PERIBÁÑEZ, M. A.; et al. Fleas parasitizing domestic dogs in Spain. Veterinary Parasitology, v.151, n.2-4, p.312-319. 2008. Disponível em: <https://doi.org/10.1016/j.vetpar.2007.10.006>. doi: 10.1016/j.vetpar.2007.10.006

HOOGLAND, J. L. Why do female Gunnison's prairie dogs copulate with more than one male? Animal Behaviour, v.55, n.2, p.351-359, 1998. Disponível em:< https://doi.org/10.1006/anbe.1997.0575>. doi: 10.1006/anbe.1997.0575.

LEVINSON, W. Microbiologia Médica e Imunologia. $12^{\mathrm{a}}$ ed. Porto Alegre: Artmed, 2014. 720p.

MATOS, M.; ALHO, A. M.; OWEN, S. P.; NUNES, T.; CARVALHO, L. M. Parasite control practices and public perception of parasitic diseases: A survey of dog and cat owners. Preventive Veterinary Medicine, v. 122, n. 1-2, p. 174-180, 2015. 
Disponível em: $\quad$ ehttps://doi.org/10.1016/j.prevetmed.2015.09.006>. doi:10.1016/j.prevetmed.2015.09.006

MELO, A. L. T.; WITTER, R.; MARTINS, T. F.; PACHECO, T. A.; ALVES, A. S.; et al. A survey of tick-borne pathogens in dogs and their ticks in the Pantanal biome, Brazil. Medical and Veterinary Entomology, v.30, n. 1, p.112-116, 2016. Disponível em:<http://onlinelibrary.wiley.com/doi/10.1111/mve.12139/full>. doi: $10.1111 /$ mve. 12139

PAZ, G. F.; AVELAR, D. M.; REIS, I. A.; LINARDI, P. M. Dynamics of Ctenocephalides felis felis (Siphonaptera: Pulicidae) Infestations on Urban Dogs in Southeastern Brazil. Journal of Medical Entomology, v. 52, n. 5, p. 1159-1164, 2015. Disponível em: <https://doi.org/10.1093/jme/tjv071>. doi: 10.1093/jme/tjv071.

RIBEIRO, C. M.; MATOS, A. C.; AZZOLINI, T.; BONES, E. R.; WASNIESKI, E. A.; et al. Molecular epidemiology of Anaplasma platys, Ehrlichia canis and Babesia vogeli in stray dogs in Paraná, Brazil. Pesquisa Veterinária Brasileira, v.37, n. 2, p.128136, 2017.2 Disponível em: <http://www.scielo.br/scielo.php?script=sci_arttext\&pid=S010036X2017000200129\&I ng=en\&nrm=iso >. doi: 10.1590/s0100-736x2017000200006.

RODRIGUES, D. F.; DAEMON, E.; RODRIGUES, A. F. Caracterização da população de ectoparasitos em cães de núcleos de expansão urbana de Juiz de Fora, Minas Gerais, Brasil. Revista Brasileira de Parasitologia Veterinária, v.17, n.4, p.185188, 2008. Disponível em:< http://dx.doi.org/10.1590/S1984-29612008000400003>. doi: 10.1590/S1984-29612008000400003.

RUBINI, A. S.; PADUAN, K. S.; MARTINS, T. F.; LABRUNA, M. B; O'DWYER, L. H. Acquisition and transmission of Hepatozoon canis (Apicomplexa: Hepatozoidae) by the tick Amblyomma ovale (Acari: Ixodidae). Veterinary Parasitology, v. 164, n. 2-4, p.324-327, 2009. Disponível em:< https://doi.org/10.1016/j.vetpar.2009.05.009 >. doi: 10.1016/j.vetpar.2009.05.009.

SOUSA, K. C. M.; FRENANDES, M. P.; HERRERA, H. M.; BENEVENUTE, J. L.; SANTOS, F. M.; et al. Molecular detection of Hepatozoon spp. in domestic dogs and wild mammals in Southern Pantanal, Brazil with implications in the transmission route. Veterinary Parasitology, v. 237, n1, p.37-46, 2017. Disponível em:<http://dx.doi.org/10.1016/j.vetpar.2017.02.023>. doi: 10.1016/j.vetpar.2017.02.023.

TAYLOR, M. A. Parasitologia Veterinária. Rio de Janeiro: Guanabara Koogan, 2010. 742p.

TORRES, F. D.; FIGUEIREDO, L. A.; FAUSTINO, M. A. G. Ectoparasitos de cães provenientes de alguns municípios da região metropolitana do Recife, Pernambuco, Brasil. Revista Brasileira de Parasitologia Veterinária, v.13, n.4, p.151-154. 2004. Disponível em:< http://cbpv.org.br/rbpv>. 
TORRES-CHABLE, O. M.; BAAK-BAAK, C. M.; CIGARROA-TOLEDO, N.; ZARAGOZA-VERA, C. V.; ARJONA-JIMENEZ, G.; et al. First report of chewing lice Heterodoxus spiniger (Enderlein, 1909) and Trichodectes canis (De Geer, 1778) on domestic dogs at Tabasco, Mexico. Southwestern Entomologist, v. 42, n. 2, p. 409-4018, 2017. Disponível em:<https://doi.org/10.3958/059.042.0211>. doi: 10.3958/059.042.0211. 